\title{
DISCOVERY OF AN M9.5 CANDIDATE BROWN DWARF IN THE TW HYDRAE ASSOCIATION: DENIS J124514.1-442907
}

\author{
Dagny L. Looper, ${ }^{2,3}$ Adam J. Burgasser, ${ }^{4}$ J. Davy Kirkpatrick, ${ }^{5}$ And Brandon J. SwifT ${ }^{2,6}$ \\ Received 2007 August 30; accepted 2007 September 20; published 2007 October 12
}

\begin{abstract}
We report the discovery of a fifth candidate substellar system in the $~ 5-10$ Myr TW Hydrae association: DENIS J124514.1-442907. This object has a NIR spectrum remarkably similar to that of 2MASS J1139511-315921, a known TW Hydrae brown dwarf, with low surface gravity features such as a triangularshaped $\mathrm{H}$ band, deep $\mathrm{H}_{2} \mathrm{O}$ absorption, weak alkali lines, and weak hydride bands. We find an optical spectral type of M9.5 and estimate a mass of $\lesssim 24 M_{\text {Jup }}$, assuming an age of $\sim 5-10$ Myr. While the measured proper motion for DENIS J124514.1-442907 is inconclusive as a test for membership, its position in the sky is coincident with the TW Hydrae association. A more accurate proper-motion measurement, higher resolution spectroscopy for radial velocity, and a parallax measurement are needed to derive the true space motion and to confirm its membership.
\end{abstract}

Subject headings: open clusters and associations: individual (TW Hydrae) stars: individual (DENIS J124514.1-442907, 2MASS J1207334-393254, 2MASS

J1139511-315921) — stars: low-mass, brown dwarfs

Online material: color figures

\section{INTRODUCTION}

Although one of the nearest groups of newly formed stars, the TW Hydrae association (TWA, Kastner et al. 1997; $d \sim$ 20-130 pc, age 5-10 Myr; Zuckerman \& Song 2004) was only recently identified due to its low spatial density over large spatial extent $\left(\alpha: 10^{\mathrm{h}}\right.$ to $14^{\mathrm{h}}, \delta:-20^{\circ}$ to $\left.-60^{\circ}\right)$. The association is composed of 25 known systems with early-type A to earlytype $\mathrm{M}$ stellar primaries, with a high fraction of companions (0.84 \pm 0.22 ; Brandeker et al. 2003). These objects share

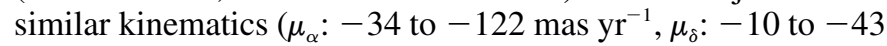
mas $\mathrm{yr}^{-1}$, and $V_{\text {rad }}:+6$ to $+17 \mathrm{~km} \mathrm{~s}^{-1}$; Reid 2003), supporting their common motion through space.

In addition, four substellar systems in TWA have been identified, two of which are not orbiting higher mass stars: 2MASSW J1139511-315921 ${ }^{7}$ and 2MASSW J1207334 $-393254 \mathrm{AB}$ (Gizis 2002), and two of which are in systems with stellar primaries: TWA $5 \mathrm{~B}$, which is a companion to TWA 5A (Lowrance et al. 1999; Webb et al. 1999), and SSSPM

\footnotetext{
${ }^{1}$ Some of the data presented herein were obtained at the W. M. Keck Observatory, which is operated as a scientific partnership among the California Institute of Technology, the University of California, and the National Aeronautics and Space Administration. The Observatory was made possible by the generous financial support of the W. M. Keck Foundation. This Letter includes data gathered with the $6.5 \mathrm{~m}$ Magellan Telescopes located at Las Campanas Observatory, Chile.

${ }^{2}$ Institute for Astronomy, University of Hawaii, 2680 Woodlawn Drive, Honolulu, HI 96822.

${ }^{3}$ Visiting Astronomer at the Infrared Telescope Facility, which is operated by the University of Hawaii under cooperative agreement NCC 5-538 with the National Aeronautics and Space Administration, Office of Space Science, Planetary Astronomy Program.

${ }^{4}$ Massachusetts Institute of Technology, Kavli Institute for Astrophysics and Space Research, Building 37, Room 664B, 77 Massachusetts Avenue, Cambridge, MA 02139; ajb@mit.edu.

${ }^{5}$ Infrared Processing and Analysis Center, M/S 100-22, California Institute of Technology, Pasadena, CA 91125.

${ }^{6}$ Currently at Steward Observatory, University of Arizona, 933 North Cherry Avenue, Tucson, AZ 85721.

${ }^{7}$ After objects are introduced they are referred to by shortened versions of their names in the form [prefix + hhmm], where "prefix" is the survey identifier name and "hhmm" is the truncated hour and minute portion of the J2000.0 coordinates.
}

J1102-3431, which might be a common-proper-motion companion to the star TW Hydrae (Scholz et al. 2005b). The substellar binary 2MASS $1207 \mathrm{AB}$ has been the subject of intense study, revealing both a disk around the primary (Sterzik et al. 2004), and possibly the secondary (Mohanty et al. 2007). 2MASS 1207A has also been confirmed as an accretor by highresolution optical spectroscopy (Mohanty et al. 2003). The B component, a late-type L dwarf, is believed to have a mass of $\sim 8 M_{\text {Jup }}$ (Chauvin et al. 2004, 2005; Mohanty et al. 2007). The identification of additional low-mass members offers prototypes at a constrained age for atmospheric studies, test beds for disk studies at the low-mass end, an opportunity to examine low-mass star formation processes in sparse associations, and excellent targets for identifying planetary-mass companions.

In this Letter, we report the discovery of a fifth candidate brown dwarf system in TWA, DENIS J124514.1-442907, identified serendipitously in the course of our spectroscopic follow-up of nearby, late-type candidates (see Burgasser et al. 2007b for a description) in the Deep Near-Infrared Survey of the Southern Sky (DENIS; Epchtein et al. 1997). We present the kinematics of DENIS 1245 in $\S 2$ and spectroscopic observations in $\S 3$, and discuss its properties in $\S 4$.

\section{KINEMATICS}

Using a linear fit to the SuperCOSMOS Sky Survey (SSS; Hambly et al. 2001a, 2001b, 2001c), DENIS, and 2MASS timeordered astrometry covering $4.1 \mathrm{yr}$ (see Table 1), we found a proper motion of $\mu_{\alpha}=-0.22^{\prime \prime} \pm 0.07^{\prime \prime} \mathrm{yr}^{-1}$ and $\mu_{\delta}=+0.02^{\prime \prime}$ $\pm 0.10^{\prime \prime}$ (not including parallactic reflex motion; see Table 2). The motion errors include an estimated uncertainty of $0.3^{\prime \prime}$ in both right ascension and declination for all three epochs.

In Table 3 we list the measured motion for DENIS 1245 and the four known TWA brown dwarf systems. While $\mu_{\alpha}$ of DENIS 1245 is in the westerly direction, it is $2.1 \sigma$ away from the mean and $1.4 \sigma$ away from the edge of the range in $\mu_{\alpha}$ of TWA stellar systems (Reid 2003). On the other hand, $\mu_{\delta}$ of DENIS 1245 is in the northerly direction, but its large error places it less than $0.5 \sigma$ away from the mean $\mu_{\delta}$ of TWA stellar systems 
TABLE 1

ASTROMETRY FOR DENIS J124514.1-442907

\begin{tabular}{|c|c|c|c|}
\hline $\begin{array}{c}\alpha \\
(\mathrm{J} 2000.0)\end{array}$ & $\begin{array}{c}\delta \\
(\mathrm{J} 2000.0)\end{array}$ & Epoch & Catalog \\
\hline 451425 & -442907.8 & 1996 Mar 27 & UKST, SSS \\
\hline $12 \quad 4514.2$ & $\begin{array}{lll}-44 & 2907\end{array}$ & 1999 May 3 & DENIS \\
\hline 124514.16 & -44 2907.7 & 2000 Apr 27 & 2MASS \\
\hline
\end{tabular}

NoTE.-Units of right ascension are hours, minutes, and seconds, and units of declination are degrees, arcminutes, and arcseconds.

(Reid 2003). Clearly, at least one more, precise astrometric measurement is needed to better constrain the motion of DENIS 1245 and to test its membership in TWA.

\section{OBSERVATIONS}

\subsection{NIR Spectroscopy}

We observed 2MASS 1139, 2MASS 1207A, ${ }^{8}$ and DENIS 1245 on three consecutive nights: 2007 March 16, 17, and 18 (UT), respectively, using the Infrared Telescope Facility's (IRTF) SpeX spectrograph (Rayner et al. 2003). Skies were clear or contained only light cirrus with $\sim 1$ " seeing at $K$. We used the prism mode with the $0.5^{\prime \prime} \times 15^{\prime \prime}$ slit (rotated to the parallactic angle), yielding a resolution of $R=\lambda / \Delta \lambda \approx 150$. 2MASS 1139 was observed at an air mass of 1.9 in four AB nodding cycles with $120 \mathrm{~s}$ of exposure time per nod position. 2MASS 1207A was observed at an air mass of 2.0 with three $\mathrm{AB}$ nodding cycles with $120 \mathrm{~s}$ of exposure time per nod position. DENIS 1245 was observed at an air mass of 2.4 with two AB nodding cycles with $120 \mathrm{~s}$ of exposure time per nod position. For flux calibration and telluric correction, the A0 V stars HD 100330 for 2MASS 1207A and HD 103870 for 2MASS 1139 and DENIS 1245 were observed at an air mass and time similar to those of the targets, followed by flat-field and argon arc lamp calibration frames. The data were reduced in standard fashion using the Spextool package version 3.2 (Cushing et al. 2004; Vacca et al. 2003).

The reduced NIR spectra are shown in Figure 1, along with the M8 standard VB 10 (J. D. Kirkpatrick et al., in preparation) and the L0 standard 2MASS J03454316+2540233 (K. L. Cruz et al., in preparation), also taken in SpeX prism mode with identical settings. DENIS 1245, like 2MASS 1139 and 2MASS 1207A, shows several hallmarks of low surface gravity, such as a triangular-shaped $H$ band; deep $\mathrm{H}_{2} \mathrm{O}$ absorption at 0.89

\footnotetext{
${ }^{8}$ Although the B component of 2MASS $1207 \mathrm{AB}$ is unresolved from the A component in our observations, it makes a negligible contribution $\left(\Delta J_{B-A} \sim\right.$ 7; Mohanty et al. 2007) to the spectrum of 2MASS 1207A. We therefore will refer to the spectroscopy of 2MASS $1207 \mathrm{AB}$ as that for 2MASS 1207A alone.
}

TABLE 2

Properties of DENIS J124514.1-442907

\begin{tabular}{|c|c|c|}
\hline Parameter & Value & Reference \\
\hline$\alpha(\mathrm{J} 2000.0)^{\mathrm{a}}$ & $12^{\mathrm{h}} 45^{\mathrm{m}} 14.20^{\mathrm{s}}$ & 1 \\
\hline$\delta(\mathrm{J} 2000.0)^{\mathrm{a}}$ & $-44^{\circ} 29^{\prime} 07.7^{\prime \prime}$ & 1 \\
\hline$\mu_{\alpha} \ldots \ldots \ldots$ & $0.22^{\prime \prime} \pm 0.07^{\prime \prime} \mathrm{yr}^{-1}$ & 2 \\
\hline$\mu_{\delta}$ & $0.02^{\prime \prime} \pm 0.10^{\prime \prime} \mathrm{yr}^{-1}$ & 2 \\
\hline$d_{\text {est }}, \ldots \ldots \ldots \ldots \ldots \ldots$ & $\sim 100 \mathrm{pc}$ & 2 \\
\hline Optical spectral type & M9.5 & 2 \\
\hline NIR spectral type ... & M9pec & 2 \\
\hline $\operatorname{Age}^{\mathrm{b}} \ldots \ldots \ldots \ldots \ldots$ & $\sim 5-10 \mathrm{Myr}$ & 2 \\
\hline $\operatorname{Mass}^{\mathrm{c}} \ldots \ldots \ldots$ & $\lesssim 24 M_{\text {Jup }}$ & 2 \\
\hline$I_{N} \ldots \ldots \ldots \ldots$ & $17.94 \mathrm{mag}$ & 3 \\
\hline 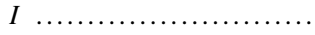 & $18.0 \pm 0.2 \mathrm{mag}$ & 1 \\
\hline ........... & $14.52 \pm 0.03 \mathrm{mag}$ & 4 \\
\hline 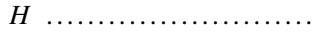 & $13.80 \pm 0.03 \mathrm{mag}$ & 4 \\
\hline 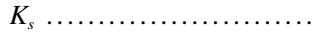 & $13.37 \pm 0.04 \mathrm{mag}$ & 4 \\
\hline$I-J \ldots \ldots+\ldots+\ldots+\cdots$ & $3.5 \pm 0.2 \mathrm{mag}$ & 1,4 \\
\hline$J-H$ & $0.72 \pm 0.04 \mathrm{mag}$ & 4 \\
\hline$H-K_{s} \ldots \ldots \ldots \ldots+\cdots \cdots$ & $0.43 \pm 0.05 \mathrm{mag}$ & 4 \\
\hline$J-K_{s} \ldots \ldots \ldots \ldots \ldots \ldots$ & $1.15 \pm 0.05 \mathrm{mag}$ & 4 \\
\hline
\end{tabular}

${ }^{a}$ Coordinates are from the DENIS Third Release Catalog at epoch 1999 May 3.

${ }^{\mathrm{b}}$ Based on age estimates of TWA (Makarov et al. 2005; de la Reza et al. 2006; Barrado y Navascués 2006).

${ }^{\mathrm{C}}$ This upper mass limit is based on the mass estimate for 2MASS 1207A (Mohanty et al. 2007).

REFERENCES. - (1) DENIS (Epchtein et al. 1997); (2) this Letter; (3) SSS (Hambly et al. 2001a, 2001b, 2001c); (4) 2MASS (Skrutskie et al. 2006).

$0.99 \mu \mathrm{m}, 1.30-1.51 \mu \mathrm{m}$, and $1.75-2.05 \mu \mathrm{m}$; and stronger VO absorption and weaker alkali absorption in comparison to field standards. Also, the FeH Wing-Ford band at $0.99 \mu \mathrm{m}$ in the spectra of 2MASS 1139, 2MASS 1207A, and DENIS 1245 is very weak in comparison to the field M8 and L0 standards.

Determining NIR spectral types of young brown dwarfs is difficult, given that the deep $\mathrm{H}_{2} \mathrm{O}$ absorption at 1.30-1.51 and $1.75-2.05 \mu \mathrm{m}$ tends to lead to later spectral typing in the NIR than in the optical. To this end, Allers et al. (2007) have created an $\mathrm{H}_{2} \mathrm{O}$ index $(1.49-1.59 \mu \mathrm{m})$, largely insensitive to surface gravity, that can be used to spectrally classify M5-L5 dwarfs in the NIR. We use this index to classify 2MASS 1207A, 2MASS 1139, and DENIS 1245 in the NIR. Overall, the NIR morphology of DENIS 1245 is nearly identical to that of 2MASS 1139, suggesting that the two have similar surface gravities.

\subsection{Optical Spectroscopy}

Red optical spectral data for DENIS 1245 were obtained on 2007 May 9 (UT) in clear conditions using the Low Dispersion Survey Spectrograph (LDSS-3; Allington-Smith et al. 1994) mounted on the Magellan $6.5 \mathrm{~m}$ Clay Telescope. Data were

TABLE 3

Spectral Types and Kinematics of TWA Brown Dwarfs

\begin{tabular}{|c|c|c|c|c|c|c|c|c|c|}
\hline Object & Optical Spectral Type & Reference & NIR Spectral Type ${ }^{a}$ & $\mathrm{H}_{2} \mathrm{O}$ Index & $\begin{array}{c}\mu_{\alpha} \\
\left(\text { mas yr }^{-1}\right)\end{array}$ & 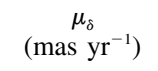 & Reference & Distance & Reference \\
\hline SSSPM 1102 & M8.5 & 1 & & $\ldots$ & $-82 \pm 12$ & $-12 \pm 6$ & 1 & $56 \pm 7$ & 1 \\
\hline TWA 5B & $\ldots$ & $\ldots$ & M8-M8.5 & $\ldots$ & $-86 \pm 8$ & $-21 \pm 8$ & 4 & $\sim 45$ & 3 \\
\hline 2MASS 1139 & M8 & 2 & M9pec ${ }^{\mathrm{c}}$ & 1.13 & $-93 \pm 5$ & $-31 \pm 10$ & 1 & $\sim 47$ & 3 \\
\hline 2MASS 1207A & M8 & 2 & M8pec ${ }^{\mathrm{c}}$ & 1.08 & $-63 \pm 3$ & $-23 \pm 2$ & 5 & $54 \pm 3$ & 5 \\
\hline DENIS 1245 & M9.5 & 3 & M9pec ${ }^{\mathrm{c}}$ & 1.14 & $-220 \pm 70$ & $+20 \pm 100$ & 3 & $\sim 100$ & 3 \\
\hline
\end{tabular}

${ }^{\text {a }}$ Derived from the $\mathrm{H}_{2} \mathrm{O}$ index (Allers et al. 2007), unless otherwise noted.

${ }^{\mathrm{b}}$ NIR photometric spectral type from Lowrance et al. (1999).

"The label "peculiar" has been appended to the NIR spectral type of these three objects based on their triangular-shaped $H$-band and other low surface gravity features (see Kirkpatrick et al. 2006).

Optical SPeCtral TYPE, KINEMATIC, AND DistanCE REFERENCES. - (1) Scholz et al. 2005b; (2) Gizis 2002; (3) this Letter; (4) Lowrance et al. 1999; (5) Gizis et al. 2007. 


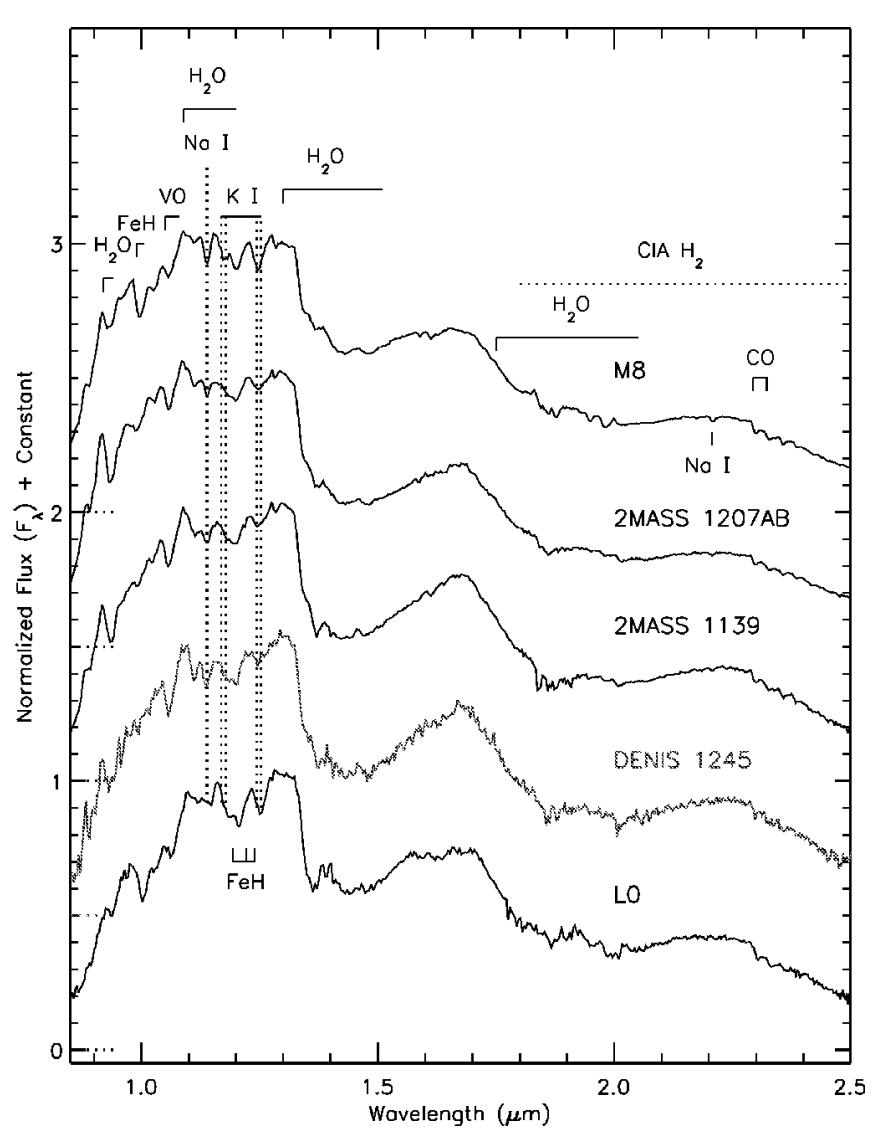

FIG. 1.-NIR spectra of DENIS 1245 (M9.5pec), 2MASS 1207A (M8pec), and 2MASS 1139 (M9pec) taken with IRTF SpeX in prism mode $(R \sim 150)$, shown in comparison to the M8 spectral standard VB 10 and the L0 spectral standard 2MASS 0345 taken with an identical setup. All spectra have been normalized at $1.27 \mu \mathrm{m}$ and are offset by half-integer values (dotted lines show zero levels) for clarity. Major atomic and molecular features have been labeled. [See the electronic edition of the Journal for a color version of this figure.]

obtained using the VPH-red grism $\left(660\right.$ lines $\left.\mathrm{mm}^{-1}\right)$ with a $0.75^{\prime \prime}$ (4 pixel) wide long slit aligned to the parallactic angle, providing $6050-10500 \AA$ spectra at a resolution $\lambda / \Delta \lambda \approx$ 1800 . The OG590 long-pass filter was used to eliminate secondorder light shortward of $6000 \AA$. A single exposure of $1500 \mathrm{~s}$ was obtained at an air mass of 1.08 , followed immediately by observation of the G2 V star HD 114853 for telluric absorption correction. Dispersion and pixel response calibration were determined by HeNeAr arc lamp and flat-field quartz lamp exposures obtained immediately after observation of both sources. The data reduction, carried out in the $\mathrm{IRAF}^{9}$ environment, is described in detail in Burgasser et al. (2007a).

The reduced red optical spectra of 2MASS 1139, 2MASS 1207A ( $R \sim 1200$; J. D. Kirkpatrick et al., in preparation), and DENIS $1245(R \sim 1800)$ are presented in Figure 2, along with the M8.5 TWA brown dwarf SSSPM $1102(R \sim 600$; Scholz et al. 2005b), the M8 comparison star 2MASS $\mathrm{J} 14342644+1940499$, and the L0 standard 2MASS 0345 $(R \sim 1200$; Kirkpatrick et al. 1999). Several features in the spectra of 2MASS 1139, 2MASS 1207A, and SSSPM 1102 supporting their low surface gravity are also seen in the spectrum of DENIS 1245. In particular, stronger VO and $\mathrm{TiO}$ absorption and weaker $\mathrm{CaH}$ and $\mathrm{K} \mathrm{I}$ absorption in comparison to the field standards. There is also no discernible FeH absorption

\footnotetext{
${ }^{9}$ IRAF is distributed by the National Optical Astronomy Observatory, which are operated by the Association of Universities for Research in Astronomy, Inc., under cooperative agreement with the National Science Foundation.
}

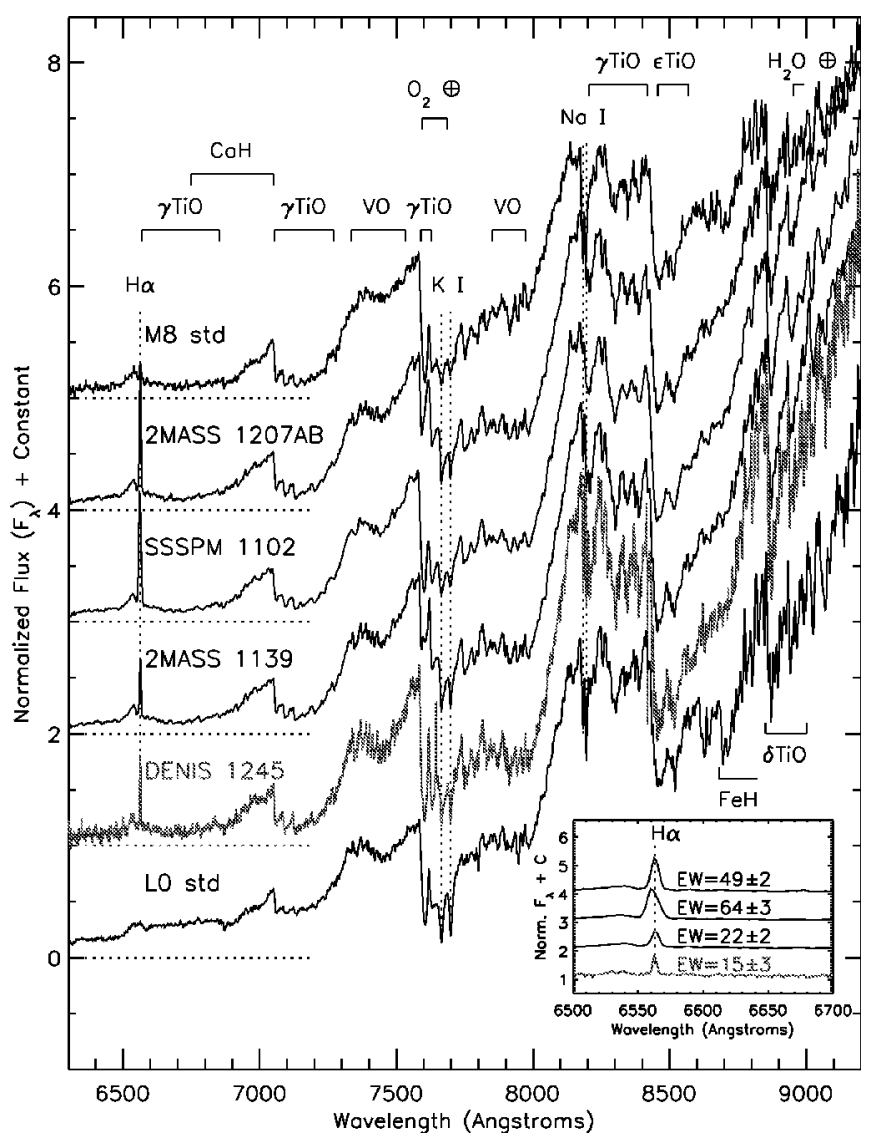

FIG. 2.-Red optical spectra of DENIS 1245 (M9.5; $R \sim 1800$ ), 2MASS 1207A, and 2MASS 1139 (M8; $R \sim 1200$ ). Shown for comparison are the M8 star 2MASS 1434, the L0 standard 2MASS $0345(R \sim 1200)$, and SSSPM 1102 (M8.5; $R \sim 600$ ). 2MASS $1207 \mathrm{AB}$, SSSPM 1102, and 2MASS 1139 are all known brown dwarf systems of TWA. All spectra have been normalized at $7500 \AA$ and are offset by integer values (dotted lines show zero levels) for clarity. Major atomic and molecular features have been labeled. The bottom right inset shows the detection of $\mathrm{H} \alpha$ emission in the TWA brown dwarfs and their measured equivalent widths (EW), following the same order as the main figure. [See the electronic edition of the Journal for a color version of this figure.]

at $8680-8820 \AA$ in the spectra of the three TWA brown dwarf systems and DENIS 1245.

The TWA brown dwarf systems and DENIS 1245 show prominent $\mathrm{H} \alpha$ emission at $6563 \AA$ (see bottom inset of Fig. 2). We have measured the EW of this feature in all four objects (see Fig. 2) using the SPLOT package in IRAF. The uncertainties in these measurements were estimated using the standard deviation of five measurements taken for each emission profile, added in quadrature to the average of five measurements of a typical noise spike in each spectrum. SSSPM 1102, which shows an asymmetric $\mathrm{H} \alpha$ profile, has the strongest measured emission, with an EW of $64 \pm 3 \AA .{ }^{10}$ DENIS 1245 has the weakest measured EW of $15 \pm 3 \AA$. It should be noted that the strength and morphology of $\mathrm{H} \alpha$ emission in the spectrum of 2MASS 1207A changes dramatically over time (Scholz et al. 2005a; Scholz \& Jayawardhana 2006). Future observations will allow us to determine whether variable emission is also present in DENIS 1245.

Since the three TWA substellar systems and DENIS 1245 become much redder than the field standards at wavelengths greater than $\sim 8500 \AA$, we have spectrally classified DENIS

\footnotetext{
${ }^{10}$ Note that Scholz et al. (2005b) measure an EW of $10 \AA$ using this same spectrum. This value was in fact a FWHM measurement and not an EW as stated (R. Scholz 2007, private communication).
} 
1245 by its morphology at wavelengths shorter than this. DENIS 1245 appears to be later in spectral type than all three TWA brown dwarf systems. We assign an optical spectral type of M9.5 based on visual comparisons to late-type M and earlytype L dwarf optical standards (see Fig. 2).

\section{DISCUSSION}

In comparison to the four known TWA brown dwarf systems, DENIS 1245 is the faintest in $J$ and also the reddest in $I-J$. These differences likely arise from DENIS 1245 being more distant and possibly cooler. Determining spectrophotometric distance estimates for young brown dwarfs is not straightforward, as well-established absolute magnitude/spectral relations based on evolved field dwarfs (e.g., Cruz et al. 2003) can significantly underestimate the distances of these overluminous, low surface gravity objects. However, Gizis et al. (2007) have recently measured the parallax of 2MASS 1207A, and we can use the absolute magnitude of this source as a proxy for DENIS 1245. Based on its $J$ magnitude and assuming similar brightness, we find $d \sim 110$ pc for DENIS 1245 . However, this estimate is likely an upper limit since DENIS 1245 has an optical spectral type 1.5 subtypes later than that of 2MASS 1207A. Cruz et al. (2003) find a $\Delta M_{J}=0.44$ mag difference between optical spectral types M8 and M9.5. If this difference were then applied to the $J$ magnitude of DENIS 1245, we would then find an estimated distance of $\sim 90 \mathrm{pc}$. So we assume a rough distance estimate of 100 pc for DENIS 1245.

Since the three other TWA brown dwarf systems have spectral types similar to that of 2MASS 1207A, we can use the parallax measurement for 2MASS 1207 A and their $J$ magnitudes to estimate distances to each of these objects (see Table 3). Scholz et al. (2005b) have suggested that SSSPM 1102 is a widely separated companion to the star TW Hydrae. Our distance estimate to SSSPM $1102(\sim 55 \mathrm{pc})$ agrees well with the distance to TW Hydrae (56 \pm 7 pc; Perryman et al. 1997).

We note that if DENIS 1245 is indeed a TWA member, its M9.5 optical spectral type implies that it is cooler and less massive than the M8 optical brown dwarf TWA 1207A $\left(T_{\text {eff }, A} \approx 2550 \pm 150 \mathrm{~K}, M_{A} \approx 24 \pm 6 M_{\text {Jup }}\right.$; Mohanty et al. 2007). This upper limit on mass $\left(M \lesssim 24 M_{\text {Jup }}\right)$ places DENIS 1245 firmly in the substellar regime.
DENIS 1245 is only the fifth substellar system to be identified within the TW Hydrae association. It was not uncovered by Gizis (2002) as it is 1 mag fainter in 2MASS $K_{s}$ than the faintest objects identified in that study with similar $J-K_{s}$ colors. Our result therefore suggests that more distant and later type substellar members of TWA may remain to be identified. Nevertheless, DENIS 1245 requires further high-resolution spectroscopic observations and a more accurate proper-motion determination to verify that it shares a common space motion with TWA. These observations, coupled with mid-infrared photometry, can also test whether this source, like 2MASS 1207A (and possibly B), harbors a disk. Lastly, as a very young, latetype brown dwarf, DENIS 1245 presents an excellent target for high-resolution imaging for planetary/substellar companions.

We thank our referee S. Mohanty for suggestions which significantly improved the manuscript. D. L. L. thanks M. Pitts for comments on the manuscript, M. Cushing for useful discussions, J. Gizis for providing an early copy of his manuscript on the parallax for 2MASS 1207A, and R. Scholz and K. Cruz for providing spectra. We also thank our telescope operators B. Golisch, D. Griep, and H. Nuñez, and instrument specialist J. Rayner for their assistance. This publication includes data from the DENIS project, which has been partly funded by the SCIENCE and the HCM plans of the European Commission under grants CT920791 and CT940627. This publication makes use of data products from the Two Micron All Sky Survey (2MASS), which is a joint project of the University of Massachusetts and the Infrared Processing and Analysis Center/ California Institute of Technology, funded by the National Aeronautics and Space Administration and the National Science Foundation. This research has made use of the NASA/IPAC Infrared Science Archive (IRSA), which is operated by the Jet Propulsion Laboratory, California Institute of Technology, under contract with the National Aeronautics and Space Administration. This research used the facilities of the Canadian Astronomy Data Centre operated by the National Research Council of Canada with the support of the Canadian Space Agency.

Facilities: Keck:I, Magellan:Clay, IRTF

\section{REFERENCES}

Allers, K. N., et al. 2007, ApJ, 657, 511

Allington-Smith, J., et al. 1994, PASP, 106, 983

Barrado y Navascués, D. 2006, A\&A, 459, 511

Brandeker, A., Jayawardhana, R., \& Najita, J. 2003, AJ, 126, 2009

Burgasser, A. J., Cruz, K. L., \& Kirkpatrick, J. D. 2007a, ApJ, 657, 494

Burgasser, A. J., Looper, D. L., Kirkpatrick, J. D., Cruz, K. L., \& Swift, B. J. 2007b, preprint (arXiv:0710.1123)

Chauvin, G., et al. 2004, A\&A, 425, L29 2005, A\&A, 438, L25

Cruz, K. L., Reid, I. N., Liebert, J., Kirkpatrick, J. D., \& Lowrance, P. J. 2003, AJ, 126, 2421

Cushing, M. C., Vacca, W. D., \& Rayner, J. T. 2004, PASP, 116, 362

de la Reza, R., Jilinski, E., \& Ortega, V. G. 2006, AJ, 131, 2609

Epchtein, N., et al. 1997, Messenger, 87, 27

Gizis, J. E. 2002, ApJ, 575, 484

Gizis, J. E., Jao, W.-C., Subasavage, J. P., \& Henry, T. J. 2007, ApJL, in press (arXiv:0709.1178)

Hambly, N. C., Davenhall, A. C., Irwin, M. J., \& MacGillivray, H. T. 2001a, MNRAS, 326, 1315

Hambly, N. C., Irwin, M. J., \& MacGillivray, H. T. 2001b, MNRAS, 326, 1295

Hambly, N. C., et al. 2001c, MNRAS, 326, 1279

Kastner, J. H., et al. 1997, Science, 277, 67
Kirkpatrick, J. D., et al. 1999, ApJ, 519, 802 . 2006, ApJ, 639, 1120

Lowrance, P. J., et al. 1999, ApJ, 512, L69

Makarov, V. V., Gaume, R. A., \& Andrievsky, S. M. 2005, MNRAS, 362, 1109

Mohanty, S., Jayawardhana, R., \& Barrado y Navascués, D. 2003, ApJ, 593, L109

Mohanty, S., Jayawardhana, R., Huélamo, N., \& Mamajek, E. 2007, ApJ, 657, 1064

Perryman, M. A. C., et al. 1997, A\&A, 323, L49

Rayner, J. T., et al. 2003, PASP, 115, 362

Reid, N. 2003, MNRAS, 342, 837

Scholz, A., \& Jayawardhana, R. 2006, ApJ, 638, 1056

Scholz, A., Jayawardhana, R., \& Brandeker, A. 2005a, ApJ, 629, L41

Scholz, R.-D., McCaughrean, M. J., Zinnecker, H., \& Lodieu, N. 2005b, A\&A, 430, L49

Skrutskie, M. F., et al. 2006, AJ, 131, 1163

Sterzik, M. F., Pascucci, I., Apai, D., van der Bliek, N., \& Dullemond, C. P. 2004, A\&A, 427, 245

Vacca, W. D., Cushing, M. C., \& Rayner, J. T. 2003, PASP, 115, 389

Webb, R. A., et al. 1999, ApJ, 512, L63

Zuckerman, B., \& Song, I. 2004, ARA\&A, 42, 685 